06

\title{
Короткие импульсы нормальных мод электромагнитно
} индуцированной прозрачности

\author{
(C) O.М. Паршков \\ Саратовский государственный технический университет имени Ю.А. Гагарина, \\ 410054 Саратов, Россия \\ e-mail: oparshkov@mail.ru
}

Поступила в редакцию 11.10 .2018 г.

В окончательной редакции 11.10.2018 г.

Принята к публикации 11.12.2018 г.

\begin{abstract}
Теоретически проанализирован процесс распространения коротких пробных импульсов электромагнитно индуцированной прозрачности при эллиптической поляризации управляющего излучения. В качестве модели резонансной среды используется $\Lambda$-схема квантовых переходов между вырожденными уровнями ${ }^{3} P_{0},{ }^{3} P_{1}^{0}$ и ${ }^{3} P_{2}$ изотопа ${ }^{208} \mathrm{~Pb}$. Изучена ситуация, когда пробное излучение достаточно слабо по сравнению с управляющим. В этом случае поле пробного импульса представимо в виде суммы полей двух эллиптически поляризованных импульсов, распространяющихся в среде независимо друг от друга без изменения состояния поляризации, что позволяет трактовать их как нестационарные нормальные моды. Численное моделирование показало, что структура нормальных мод зависит от отношения ширины спектра входного пробного импульса к ширине спектра допплеровского разброса частот квантового перехода, резонансного пробному полю. При малой величине этого отношения каждая мода в среде представляет собой моноимпульс, подобный входному пробному импульсу. В этих условиях перемещение каждой нормальной моды в среде может быть достаточно хорошо охарактеризовано групповой скоростью, зависящей от интенсивности и состояния поляризации управляющего излучения. При увеличении указанного соотношения нормальные моды в среде приобретают сначала вид регулярного затухающего цуга импульсов, а затем их структура становится хаотической. Описанная эволюция сопровождается увеличением поглощения энергии пробного излучения средой и существенным ухудшением применимости понятия групповой скорости для описания процесса распространения нормальных мод.
\end{abstract}

DOI: $10.21883 /$ OS.2019.04.47520.304-18

\section{1. Введение}

Явление взаимного гашения амплитуд вероятностей квантовых переходов между энергетическими уровнями при резонансном возбуждении среды многочастотным когерентным лазерным излучением представляет значительный теоретический и практический интерес. Это явление лежит в основе таких эффектов, как пленение населенности $[1,2]$ и электромагнитно индуцированная прозрачность (ЭМИП) [3-5]. В частности, в связи с эффектом ЭМИП отметим, что его использование перспективно для создания систем оптической квантовой памяти [4], систем квантовых коммуникаций $[4,6,7]$ и квантовой информации [3-5], устройств точных магнитных измерений [8] и хронометрии [9]. Эффект ЭМИП лежит в основе методов создания больших оптических нелинейностей $[5,10]$ и усиления излучения без инверсии населенностей [11]. Продолжается активное теоретическое и экспериментальное изучение специфики ЭМИП в различных ситуациях. Так, например, исследуются особенности этого явления в сильно коррелированных квантовых газах [12], в радиодиапазоне [13], на примесях в фотонных кристаллах [14], в присутствии нановолокна [15].

Явление ЭМИП при наличии вырождения энергетических уровней квантовых переходов приводит к ря- ду эффектов, связанных с поляризационными характеристиками взаимодействующих излучений. В $[16,17]$ теоретически и экспериментально исследовалось сопровождающее ЭМИП вращение плоскости поляризации пробного поля при изменении напряженности управляющего излучения, а в $[18,19]$ теоретически и экспериментально изучалось влияние постоянного магнитного поля на процесс эволюции круговых компонент пробного излучения. Линейное и круговое двойное лучепреломление пробного поля при ЭМИП исследовалось теоретически и экспериментально в [20]. В теоретической работе [21] предсказана возможность вращения плоскости поляризации линейно поляризованного входного пробного импульса и разбиение его на импульсы с взаимно перпендикулярными линейными поляризациями.

В работе [22] теоретически рассматривалось явление ЭМИП в $\Lambda$-схеме квантовых переходов между вырожденными уровнями ${ }^{3} P_{0},{ }^{3} P_{2},{ }^{3} P_{1}^{0}$ изотопа ${ }^{208} \mathrm{~Pb}$, в парах которого экспериментально наблюдалась ЭМИП поляризованных по кругу лазерных полей [23,24]. Было показано, что в случае, когда пробное поле слабо по сравнению с управляющим, и оба поля на входе в среду эллиптически поляризованы, в среде возникает двойное лучепреломление с эллиптически поляризованными нормальными модами пробного излучения. Если входное 
пробное поле представляет собой достаточно короткий импульс, то нормальные моды в среде также являлись импульсами, распространяющимися с различными групповыми скоростями. Это приводит к распаду пробного импульса на две части, каждая из которых является нормальной модой.

Цель представленной работы заключается в описании зависимости характеристик нормальных мод пробного поля от длительности импульса входного пробного излучения. В частности, будут рассмотрены вопросы влияния указанной длительности на затухание нормальных мод в среде и возможность применения к ним понятия групповой скорости.

\section{2. Нормальные моды пробного излучения}

\section{Нормальные моды пробного излучения внутри среды}

Изучаемая $\Lambda$-схема (рис. 1$)$ состоит из невырожденного нижнего $\left({ }^{3} P_{0}\right)$, пяти- и трехкратно вырожденных среднего $\left({ }^{3} P_{2}\right)$ и верхнего $\left({ }^{3} P_{1}^{0}\right)$ уровней изотопа ${ }^{208} \mathrm{~Pb}$. Пусть $\phi_{k}(k=1,2, \ldots, 9)$ - ортонормированный базис из общих собственных функций операторов энергии, квадрата момента импульса и его проекции на ось $z$ для изолированного атома, соответствующих нижнему $(k=1 ; M=0)$, верхнему $(k=2,3,4 ; M=-1,0,1)$ и среднему $(k=5,6, \ldots, 9 ; M=-2,-1,0,1,2)$ уровням. Обозначим через $D_{1}$ и $D_{2}$ приведенные электродипольные моменты переходов ${ }^{3} P_{0} \rightarrow{ }^{3} P_{1}^{0}$ и ${ }^{3} P_{2} \rightarrow{ }^{3} P_{1}^{0}$ соответственно, а через $\omega_{1}$ и $\omega_{2}\left(\omega_{1}>\omega_{2}\right)$ - центральные частоты этих переходов (рис. 1). Для учета допплеровского уширения спектральных линий введем время неоднородного уширения $T_{1}$, определяемое формулой

$$
T_{1}=\left(c / \omega_{1}\right) \sqrt{M} / \sqrt{2 k T},
$$

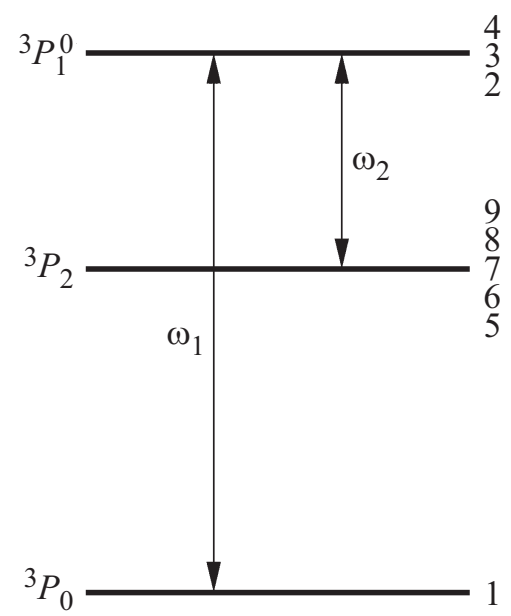

Рис. 1. $\Lambda$-схема квантовых переходов; числа справа - номера состояний в математической модели. где $M$ - масса атома ${ }^{208} \mathrm{~Pb}, T$ - абсолютная температура его паров, $k$ - постоянная Больцмана. Ширина $\Delta_{1}$ (по уровню $e^{-1}$ от максимума) контура линии неоднородного уширения перехода ${ }^{3} P_{0} \rightarrow{ }^{3} P_{1}^{0}$ связана с величиной $T_{1}$ соотношением $\Delta_{1}=2 / T_{1}$.

Положим, что полное электрическое поле, создаваемое двумя лазерными излучениями, распространяющимися вдоль оси $z$, имеет вид

$$
\begin{gathered}
\mathbf{E}=\mathbf{E}_{1}+\mathbf{E}_{2}, \\
\mathbf{E}_{l}=\mu_{l}\left[\mathbf{i} E_{x l} \cos \left(\omega_{l} t-k_{l} z+\delta_{x l}\right)\right. \\
\left.+\mathbf{j} E_{y l} \cos \left(\omega_{l} t-k_{l} z+\delta_{y l}\right)\right], \\
l=1,2,
\end{gathered}
$$

где $\mathbf{E}_{l}$ и $\omega_{l}-$ напряженность электрического поля и несущая частота пробного $(l=1)$ и управляющего $(l=2)$ полей, $\mu_{l}=\hbar \sqrt{2 l+1} /\left(\left|D_{l}\right| T_{1}\right), \mathbf{i}, \mathbf{j}$ - единичные векторы осей $x$ и $y, E_{x l}, E_{y l}$ - неотрицательные действительные амплитуды, а $\delta_{x l}, \delta_{y l}-$ фазовые добавки $x$ и $y$-компонент пробного $(l=1)$ и управляющего $(l=2)$ полей, $k_{l}=\omega_{l} / c$. Величины $E_{x l}, E_{y l}, \delta_{x l}, \delta_{y l}$ являются функциями от $z$ и $t$. Определим переменные $f_{l}$ и $g_{l}$ равенствами

$$
\begin{gathered}
f_{l}=\left[E_{x l} \exp \left(i \delta_{x l}\right)-i E_{y l} \exp \left(i \delta_{y l}\right)\right] / \sqrt{2}, \\
g_{l}=\left[E_{x l}\left(i \delta_{x l}\right)+i E_{y l} \exp \left(i \delta_{y l}\right)\right] / \sqrt{2} .
\end{gathered}
$$

Следуя [25], именуем $f_{l}$ и $g_{l}$ амплитудами левой и правой круговых компонент пробного $(l=1)$ и управляющего $(l=2)$ полей. Волновую функцию $\Psi$ атома в поле (1) представим в виде разложения по базису $\phi_{k}$ $(k=1,2, \ldots, 9)$ :

$$
\begin{aligned}
\Psi=\bar{c}_{1} \phi_{1} & +\left(\sum_{k=2}^{4} \bar{c}_{k} \phi_{k}\right) \exp \left(-i \xi_{1}\right) \\
& +\left(\sum_{k=5}^{9} \bar{c}_{k} \phi_{k}\right) \exp \left[-i\left(\xi_{1}-\xi_{2}\right)\right],
\end{aligned}
$$

где $\bar{c}_{k}(k=1,2, \ldots, 9)$ - амплитуды вероятности заселения квантовых состояний, $\xi_{l}=\omega_{l} t-k_{l} z, l=1,2$. Введем величины $c_{i}$ следующими соотношениями:

$$
\begin{gathered}
c_{1}=p_{1}^{*} \bar{c}_{1}, \quad c_{2}=\bar{c}_{2}, \quad c_{4}=\bar{c}_{4}, \quad c_{5}=p_{2} \bar{c}_{5}, \\
c_{7}=(1 / \sqrt{6}) p_{2} \bar{c}_{7}, \quad c_{9}=p_{2} \bar{c}_{9},
\end{gathered}
$$

где $p_{l}=2 D_{l} /\left|D_{l}\right|, l=1,2$. Определим нормированные независимые переменные $s$ и $w$ :

$$
s=z / z_{0}, \quad w=(t-z / c) / T_{1},
$$

где $z_{0}=3 \hbar c /\left(2 \pi N\left|D_{1}\right|^{2} T_{1} \omega_{1}\right), N-$ концентрация атомов.

Ниже используются параметры $a_{l}, \alpha_{l}, \gamma_{l}$ эллипса поляризации (ЭП) пробного $(l=1)$ и управляющего $(l=2)$ 
излучений. Здесь $a_{l}-$ большая полуось ЭП, измеренная в единицах $\mu_{l}, \alpha_{l}-$ угол ее наклона к оси $x$ (в радианах), $\gamma_{l}$ - параметр сжатия $\left(0 \leq \alpha_{l}<\pi,-1 \leq \gamma_{l} \leq+1\right)$ [26]. Величина $\left|\gamma_{l}\right|$ определяет отношение малой оси ЭП к его большой оси. Отрицательные значения величины $\gamma_{l}$ соответствуют правой эллиптической поляризации излучения, положительные - левой.

Будем полагать, что пробное поле настолько слабо по сравнению с управляющим, что его влияние может быть учтено в рамках метода последовательных приближений по степеням малого параметра

$$
\tilde{v}=\sqrt{E_{x l}^{2}+E_{y l}^{2}} / \sqrt{E_{x 2}^{2}+E_{y 2}^{2}} .
$$

Нулевое приближение этого метода предполагает, что $\tilde{v}=0$, т.е. что пробное поле отсутствует. Поскольку энергетические уровни квантовых переходов, резонансных управляющему полю, первоначально не заселены, то это поле не взаимодействует со средой в нулевом приближении, так что в этом приближении

$$
f_{2}(w, s)=f_{20}(w), \quad g_{2}(w, s)=g_{20}(w),
$$

где $f_{20}(w)$ и $g_{20}(w)$ - круговые компоненты управляющего поля на входе в среду. Более того, будем полагать, что $f_{20}(w)=f_{20}, g_{20}(w)=g_{20}$, где $f_{20}$ и $g_{20}$ - постоянные величины. Эти условия соответствует схеме контринтуитивного наложения полей [3], которая наиболее часто используется при экспериментальном изучении ЭМИП. Тогда уравнения эволюции пробного поля в первом порядке метода последовательных приближений примут [22] следующий вид:

$$
\begin{gathered}
\frac{\partial f_{1}}{\partial s}=\frac{2 i}{\sqrt{\pi}} \int_{-\infty}^{+\infty} c_{2}^{*} \exp \left(-\varepsilon_{1}^{2}\right) d \varepsilon_{1}, \\
\frac{\partial g_{1}}{\partial s}=-\frac{2 i}{\sqrt{\pi}} \int_{-\infty}^{+\infty} c_{4}^{*} \exp \left(-\varepsilon_{1}^{2}\right) d \varepsilon_{1}, \\
\frac{\partial c_{2}}{\partial w}+i \varepsilon_{1} c_{2}=-\frac{i}{2} f_{1}^{*}-\frac{i}{4} g_{20}^{*}\left(c_{5}-\kappa_{2} c_{7}\right)-\gamma c_{2}, \\
\frac{\partial c_{4}}{\partial w}+i \varepsilon_{1} c_{4}=\frac{i}{2} g_{1}^{*}-\frac{i}{4} g_{20}^{*}\left(c_{7}-\kappa_{2} c_{9}\right)-\gamma c_{4}, \\
\frac{\partial c_{5}}{\partial w}+i\left(\varepsilon_{1}-\varepsilon_{2}\right) c_{5}=-i g_{20} c_{2}, \\
\frac{\partial c_{7}}{\partial w}+i\left(\varepsilon_{1}-\varepsilon_{2}\right) c_{7}=\frac{i}{6} g_{20}\left(\kappa_{2} c_{2}-c_{4}\right), \\
\frac{\partial c_{9}}{\partial w}+i\left(\varepsilon_{1}-\varepsilon_{2}\right) c_{9}=\frac{i}{6} \kappa_{2} g_{20} c_{2},
\end{gathered}
$$

где

$$
\begin{gathered}
\varepsilon_{1}=\left(\omega_{1}^{\prime}-\omega_{1}\right) / \Delta_{1}, \quad \varepsilon_{2}=\left(\omega_{2} / \omega_{1}\right) \varepsilon_{1}, \\
\kappa_{2}=\left(1+\gamma_{2}\right) /\left(1-\gamma_{2}\right) .
\end{gathered}
$$

Уравнения (3) справедливы при условии, что $\alpha_{2}=0$, т.е. большая ось ЭП управляющего поля совпадает с осью $x$.
Система уравнений (3) не содержит амплитуд $\bar{c}_{2}, \bar{c}_{6}$ и $\bar{c}_{8}$, что согласуется с правилами отбора $(\Delta M= \pm 1)$ для переходов под действием круговых компонент поля (1). В уравнения для $c_{2}$ и $c_{4}$ феноменологически введены слагаемые $-\gamma c_{2}$ и $-\gamma c_{4}$ для учета спонтанного распада состояний верхнего уровня рассматриваемой $\Lambda$-схемы. Здесь $\gamma=T_{1} /(2 \tau)$, где $\tau$ - радиационное время жизни уровня ${ }^{6} P_{1}^{0}$.

Согласно [27], для выбранных переходов ${ }^{208} \mathrm{~Pb}$ $\omega_{2} / \omega_{1}=0.7$ и (при $\left.T=900-1000 \mathrm{~K}\right) \gamma=1.5 \cdot 10^{-2}$. В случае $T=950 \mathrm{~K}$ имеем $T_{1}=1.63 \cdot 10^{-10} \mathrm{~s}$. Выбирая для оценок насыщенные пары ${ }^{208} \mathrm{~Pb}$ и используя данные [28], находим при той же температуре $N=3.4 \cdot 10^{13} \mathrm{~cm}^{-3}$ и $z_{0}=0.03 \mathrm{~cm}$.

Введем в рассмотрение два пробных поля с напряженностями $\mathbf{E}_{1}^{(1)}$ и $\mathbf{E}_{1}^{(2)}$. Поле $\mathbf{E}_{1}^{(1)}$ описывает эллиптически поляризованный пробный импульс со следующими характеристиками:

$$
\begin{gathered}
\alpha_{1}=\alpha_{1}^{(1)}=0, \quad a_{1}=a_{1}^{(1)}, \\
\gamma_{1}=\gamma_{1}^{(1)}, \quad \delta_{x 1}=\delta_{x 1}^{(1)},
\end{gathered}
$$

где $a_{1}^{(1)}$ и $\delta_{x 1}^{(1)}$ могут зависеть от $w$ и $s$, тогда как $\gamma_{1}^{(1)}$ является постоянной величиной. Этот импульс будем называть параллельной модой, так как главная ось его ЭП параллельна главной оси ЭП управляющего поля. Поле $\mathbf{E}_{1}^{(2)}$ описывает эллиптически поляризованный пробный импульс, для которого

$$
\begin{gathered}
\alpha_{1}=\alpha_{1}^{(2)}=\pi / 2, \quad a_{1}=a_{1}^{(2)}, \\
\gamma_{1}=\gamma_{1}^{(2)}=-\gamma_{1}^{(1)}, \quad \delta_{x 1}=\delta_{x 1}^{(2)},
\end{gathered}
$$

причем $a_{1}^{(2)}$ и $\delta_{x 1}^{2}$ могут зависеть от $w$ и $s$, тогда как $\gamma_{1}^{(2)}$ постоянна. Этот импульс будем называть перпендикулярной модой, поскольку главная ось его ЭП перпендикулярна главной оси ЭП управляющего излучения. Эксцентриситеты ЭП мод одинаковы, а направления вращения электрических векторов взаимно противоположны. Используя (2), можно показать [22], что если $f_{1}^{(i)}$ и $g_{1}^{(i)}-$ комплексные амплитуды лево и право поляризованной круговых компонент параллельной $(i=1)$ и перпендикулярной $(i=2)$ мод, то

$$
f_{1}^{(1)}=\kappa g_{1}^{(1)}, \quad f_{1}^{(2)}=-\frac{1}{\kappa} g_{1}^{(2)}, \quad \kappa=\frac{1+\gamma_{1}^{(2)}}{1-\gamma_{1}^{(1)}} .
$$

Векторы Джонса параллельной и перпендикулярной мод взаимно ортогональны, и поэтому напряженность $\mathbf{E}_{1}$ пробного поля может быть представлена в виде $\mathbf{E}_{1}=\mathbf{E}_{1}^{(1)}+\mathbf{E}_{1}^{(2)}[25]$.

В [22] показано, что если величина $\kappa$ определяется соотношениями

$$
\kappa=-p+\sqrt{p^{2}+1}, \quad p=10 \gamma_{2} /\left(1-\gamma_{2}^{2}\right),
$$


то эволюция величины $g_{1}^{(1)}$ описывается следующей системой уравнений:

$$
\begin{gathered}
\frac{\partial g_{1}^{(1)}}{\partial s}=\frac{2 i}{\sqrt{\pi}} \int_{-\infty}^{+\infty} U_{1}^{*} \exp \left(-\varepsilon_{1}^{2}\right) d \varepsilon_{1}, \\
\frac{\partial U_{1}}{\partial w}+i \varepsilon_{1} U_{1}=-\frac{i}{2} g_{1}^{(1) *}-\frac{i}{4} g_{20}^{*} V_{1}-\gamma U_{1}, \\
\frac{\partial V_{1}}{\partial w}+i\left(\varepsilon_{1}-\varepsilon_{2}\right) V_{1}=-i g_{20} q_{1} U_{1} .
\end{gathered}
$$

Входящие в (8) величины $U_{1}, V_{1}$ и $q_{1}$ определяются формулами

$$
\begin{gathered}
U_{1}=\frac{1}{\kappa^{2}+1}\left(\kappa c_{2}-c_{4}\right), \\
V_{1}=\frac{1}{\kappa^{2}+1}\left[\kappa c_{5}-\left(\kappa_{2} \kappa+1\right) c_{7}+\kappa_{2} c_{9}\right], \\
q_{1}=1+\kappa_{2}\left[\kappa_{2}+(1 / \kappa)\right] / 6 .
\end{gathered}
$$

В свою очередь, согласно [22] эволюция величины $g_{1}^{(2)}$ определяется следующей системой уравнений:

$$
\begin{gathered}
\frac{\partial g_{1}^{(2)}}{\partial s}=\frac{2 i}{\sqrt{\pi}} \int_{-\infty}^{+\infty} U_{2}^{*} \exp \left(-\varepsilon_{1}^{2}\right) d \varepsilon_{1}, \\
\frac{\partial U_{2}}{\partial w}+i \varepsilon_{1} U_{2}=-\frac{i}{2} g_{1}^{(2) *}-\frac{i}{4} g_{20}^{*} V_{2}-\gamma U_{2}, \\
\frac{\partial V_{2}}{\partial w}+i\left(\varepsilon_{1}-\varepsilon_{2}\right) V_{2}=-i g_{20} q_{2} U_{2}
\end{gathered}
$$

где

$$
\begin{gathered}
U_{2}=-\frac{\kappa^{2}}{\kappa^{2}+1}\left(\frac{1}{\kappa} c_{2}+c_{4}\right), \\
V_{2}=\frac{\kappa^{2}}{\kappa^{2}+1}\left(\frac{\kappa_{2}-\kappa}{\kappa} c_{7}-\frac{1}{\kappa_{2}} c_{5}+\kappa_{2} c_{9}\right), \\
q_{2}=1+\kappa_{2}\left(\kappa_{2}-\kappa\right) / 6 .
\end{gathered}
$$

Системы уравнений (8) и (9) не содержат общих зависимых переменных и, следовательно, могут решаться независимо друг от друга. Это означает, что при выполнении условия (7) параллельная и перпендикулярная моды пробного поля распространяются в среде независимо друг от друга. При этом согласно (4) и (5) поляризационные характеристики $\alpha_{1}^{(l)}$ и $\gamma_{1}^{(l)}$ этих мод остаются неизменными. Сказанное выше позволяет прийти к выводу, что перпендикулярная и параллельная моды представляют собой эллиптически поляризованные нормальные моды пробного излучения.

Учитывая (7) и определение величины $\kappa(6)$, можно найти зависимость величины параметра сжатия $\gamma_{1}^{(1)}$ ЭП параллельной нормальной моды от параметра сжатия $\gamma_{2}$ ЭП управляющего поля. График данной зависимости представлен в [22]. Здесь только отметим, что знак величины $\gamma_{1}^{(1)}$ противоположен знаку величины $\gamma_{2}$ и зависимость $\gamma_{1}^{(1)}$ от $\gamma_{2}$ описывается нечетной функцией.
При $\gamma_{2}=0$ имеем $\gamma_{1}^{(1)}=\gamma_{1}^{(2)}=0$, т. е. в случае линейной поляризации управляющего поля обе нормальные моды линейно поляризованы. Если величина $\left|\gamma_{2}\right|$ приближается к единице, то $\left|\gamma_{1}^{(1)}\right|$ также приближается к этому значению. Следовательно, при круговой поляризации пробного поля обе нормальные моды также будут поляризованы по кругу.

Если входное пробное поле, т.е. поле на входной поверхности $(s=0)$, имеет вид достаточно короткого импульса, то и в среде каждая нормальная мода будет представлять собой импульс. По мере распространения импульс, соответствующий каждой нормальной моде, деформируется и затухает. Однако в присутствии ЭМИП указанные факторы достаточно медленно проявляются с ростом расстояния, пройденного импульсом в среде. Это позволяет приближенно оценить групповые скорости распространения импульсов нормальных мод. В работе [22] оценка групповых скоростей мод была проведена для случая столь короткого пробного импульса, что ширина его спектра в среде значительно превышала допплеровскую ширину спектрального перехода, резонансного управляющему полю. В Приложении показано, что с той же степенью обоснованности эту оценку можно применить и в случае пробного импульса произвольной длительности. А именно, если $V_{1}^{(i)}-$ групповая скорость импульса параллельной $(i=1)$ и перпендикулярной $(i=2)$ нормальных мод в системе отсчета $s, w$, то

$$
V_{1}^{(i)}=\left(\frac{1}{8} I_{2}\right) \frac{\left(1-\gamma_{2}\right)^{2}}{1+\gamma_{2}^{2}} q_{i}, \quad i=1,2,
$$

где $I_{2}$ - интенсивность (плотность потока энергии поля в расчете на единицу площади поперечного сечения) управляющего излучения. Из (10) вытекает, что $V_{1}^{(i)}$ пропорционально $I_{2}$ и зависит от $\gamma_{2}$ - параметра сжатия ЭП управляющего излучения.

\section{Нормальные моды пробного излучения на входной поверхности среды}

Нормальные моды возникают при распространении в среде пробного излучения, возбуждаемого входным пробным импульсом с произвольными поляризационными характеристиками. Будем называть ниже это излучение порождающим. Полагая, что на входе в резонансную среду порождающее поле поляризовано эллиптически, введем обозначения $a_{1}, \alpha_{1}, \gamma_{1}, \delta_{x 1}$ для величин, описывающих это поле при $s=0$. Ограничиваясь также случаем отсутствия фазовой модуляции порождающего излучения на входной поверхности без дальнейшего ограничения общности теории, можно положить $\delta_{x 1}=0$. Характеристики (4) и (5) нормальных мод на входной поверхности должны быть такими, чтобы сумма полей мод совпадала на этой поверхности с полем входного порождающего излучения. Эти характеристики используются как граничные условия при решении 
систем уравнений (8) и (9). Формулы, определяющие характеристики нормальных мод на входной поверхности среды, приведены в [22], и здесь обсуждаться не будут.

\section{3. Эволюция нормальных мод в среде: численные результаты}

Предположим, что эллиптически поляризованное управляющее поле в среде имеет следующие поляризационные и фазовые характеристики:

$$
\begin{gathered}
\alpha_{2}=0, \quad a_{2}=6.65, \\
\gamma_{2}=-0.3, \quad \delta_{x 2}=0 .
\end{gathered}
$$

Выбор нулевого значения величины $\alpha_{2}$ диктуется изложенной выше теорией и не ограничивает общности результатов. Выбор величин $a_{2}$ и $\gamma_{2}$ означает, что при температуре насыщенного пара ${ }^{208} \mathrm{~Pb}$, равной $950 \mathrm{~K}$, интенсивность управляющего поля во время нахождения в среде пробного излучения составляет около $20 \mathrm{~kW} / \mathrm{cm}^{2}$. Это примерно соответствует условиям экспериментов, описанных в работах $[23,24]$. Постоянство величины $\delta_{x 2}$ означает отсутствие фазовой модуляции управляющего поля. Подробное обоснование выбора характеристик пробного и управляющего излучений на входе в среду, а также параметров квантовых переходов и резонансной среды содержатся в работе [21].

Нормальные моды пробного излучения возникают в среде при падении на входную поверхность импульса порождающего излучения. Положим, что этот импульс имеет следующие характеристики:

$$
\begin{gathered}
\alpha_{1}=\pi / 6, \quad a_{1}=0.2 \exp \left[-\left(w-w_{0}\right)^{2} / \tau_{p}^{2}\right], \\
\gamma_{1}=-0.5, \quad \delta_{x 1}=0 .
\end{gathered}
$$

Значения величин $\alpha_{1}$ и $\gamma_{1}$ постоянны и выбраны произвольно. Большая полуось ЭП импульса меняется во времени по закону, описываемому функцией Гаусса с временным параметром $\tau_{p}$, имеющим смысл половины длительности импульса по уровню $e^{-1}$ максимума в единицах измерения времени $w$. Максимальное значение этой функции более чем в 30 раз меньше величины $a_{2}$, так что пробное поле можно считать слабым по сравнению с управляющим. Величина $w_{0}$ является точкой экстремума функции Гаусса. Входной пробный импульс, так же как управляющее излучение, лишен фазовой модуляции. Введем обозначения $T_{p}$ и $\Delta_{p}$ для размерных длительности и ширины спектра Фурье импульса, описываемого функцией $a_{1}$ (по уровню $e^{-1}$ высоты). Нетрудно показать, что

$$
T_{p} / \tau=4 \gamma \tau_{p}, \quad \Delta_{p} / \Delta_{1}=2 / \tau_{p},
$$

где $\tau$ - упомянутое ранее радиационное время жизни уровня ${ }^{3} P_{1}^{0}$.
Используем описанный в [22] способ нахождения параметров нормальных мод на входной поверхности среды по известным параметрам входного пробного импульса (12) и управляющего поля (11). Тогда получим, что параллельная нормальная мода при $s=0$ описывается следующим образом:

$$
\begin{gathered}
\alpha_{1}=0, \\
a_{1}=0.0720 \exp \left[-\left(w-w_{0}\right)^{2} / \tau_{p}^{2}\right], \\
\gamma_{1}=0.7417, \quad \delta_{x 1}=-0.4993,
\end{gathered}
$$

а характеристики перпендикулярной моды при $s=0$ таковы:

$$
\begin{gathered}
\alpha_{1}=\pi / 2, \\
a_{1}=0.1645 \exp \left[-\left(w-w_{0}\right)^{2} / \tau_{p}^{2}\right], \\
\gamma_{1}=-0.07417, \quad \delta_{x 1}=0.2884 .
\end{gathered}
$$

Оценки по формулам (10) дают тогда следующие значения групповых скоростей нормальных мод: $V_{1}^{(1)}=9.9$ и $V_{1}^{(2)}=4.1$.

Ниже для четырех значений величины $\tau_{p}$ будут представлены результаты численного решения системы (3) при условиях (12), (14) и (15). Сначала положим $\tau_{p}=75$, что соответствует $T_{p}=25 \mathrm{~ns}$. Тогда согласно (13) $\Delta_{p} / \Delta_{1}=2 / 75$, т. е. спектр входного пробного импульса значительно уже спектра частот резонансного перехода для пробного поля. Кроме того, $T_{p} / \tau=4.5$, т.е. длительность входного пробного импульса значительно превосходит как время неоднородного уширения, так и время необратимой релаксации.

На рис. 2 приведены графики интенсивности $I_{1}$, измеряемой в единицах $c \mu_{1}^{2} /(8 \pi)$, а также величин $\alpha_{1}$ и $\gamma_{1}$ для порождающего излучения. Порождающий импульс на входе в среду эллиптически поляризован и в соответствии с (12) имеет постоянные значения величин $\alpha_{1}$ и $\gamma_{1}$ (рис. 2,a). Однако на достаточно большом расстоянии (рис. 2,b) этот импульс начинает распадаться, причем величины $\alpha_{1}$ и $\gamma_{1}$ существенно меняются со временем. Такое состояние поляризации поля не является эллиптическим в традиционном понимании [26]. На еще больших расстояниях (рис. 2,c,d) пробный импульс распадается на два импульса таким образом, что в области нахождения каждого из них поляризационные характеристики $\alpha_{1}$ и $\gamma_{1}$ постоянны. В частности, при $s=4000$ (рис. $2, d$ ) в области нахождения левого импульса $\alpha_{1}=0, \gamma_{1}=0.7417$, что в пределах используемой точности расчета совпадает со значением этих величин для параллельной нормальной моды. С подобной же точностью для правого импульса (рис. $2, d) \alpha_{1}=\pi / 2$, $\gamma_{1}=-0.7417$. Такие поляризационные характеристики имеет перпендикулярная нормальная мода.

На рис. 3 представлены графики величин $I_{1}, \alpha_{1}$ и $\gamma_{1}$ для нормальных мод, порождаемых описанным выше пробным излучением. При распространении нормальных мод в среде поляризационные характеристики $\alpha_{1}$ и $\gamma_{1}$ 

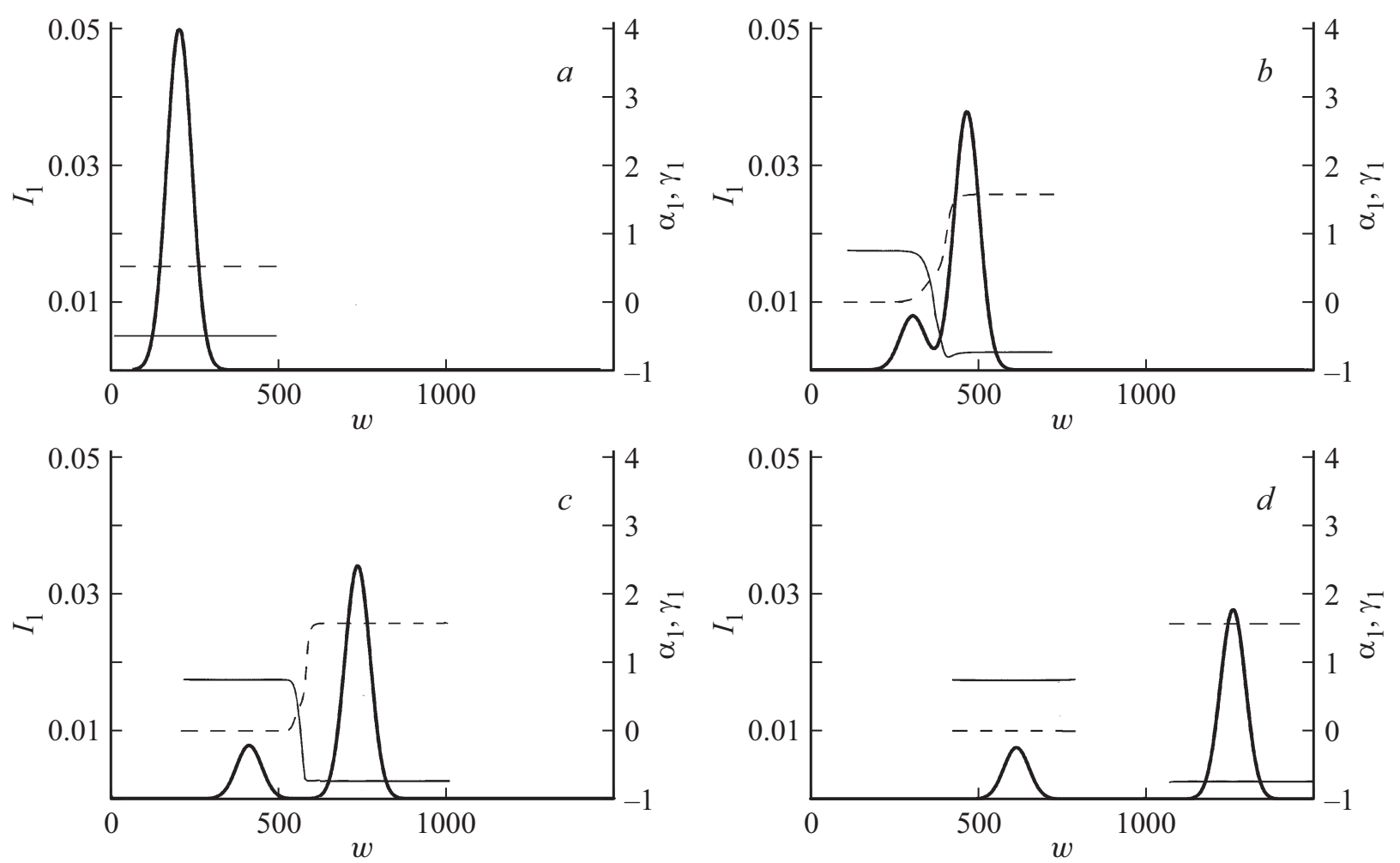

Рис. 2. Эволюция характеристик порождающего пробного поля в среде при $s=0(a), 1000(b), 2000(c), 4000(d) ; I_{1}-$ толстые линии, $\alpha_{1}-$ штрихи, $\gamma_{1}-$ тонкие линии.

остаются постоянными, и их значения такие же, как на входе в резонансную среду. Таким образом, результаты численного моделировании согласуются с результатами изложенной выше аналитической теорией нормальных мод. Кроме того, для каждой фиксированной пары значений $s, w$ с ошибкой менее $0.1 \%$ выполняется условие

$$
I_{1}=I_{1}^{(1)}+I_{1}^{(2)}
$$

где $I_{1}-$ интенсивность порождающего излучения, а $I_{1}^{(1)}$ и $I_{1}^{(2)}-$ интенсивности параллельной и перпендикулярной мод соответственно. Данное обстоятельство и постоянство величин $\alpha_{1}$ и $\gamma_{1}$ позволяют называть эти моды нормальными [25].

Расчеты и аналитическая теория показывают, что описанный выше характер эволюции поляризационных параметров $\alpha_{1}$ и $\gamma_{1}$ не зависит от конкретного значения величины $\tau_{p}$. Поэтому далее графики величин $\alpha_{1}$ и $\gamma_{1}$ не приводятся. Условие (16) также выполняется во всех рассмотренных ниже случаях.

Результаты численного моделирования позволяют заключить, что $V_{1}^{(1)}=9.6$ и $V_{1}^{(2)}=3.9$. (За групповую скорость моды принимается скорость движения в среде точки максимальной интенсивности этой моды.) Вопрос о природе расхождений этих значений и значений, предсказываемых аналитической теорией, будет обсужден позже.
Теперь положим, что $\tau_{p}=4\left(T_{p}=1.3 \mathrm{~ns}\right)$. В этом случае $\Delta_{p} / \Delta_{1}=0.5, T_{p} / \tau=0.24$. На рис. $4, a-c$ представлены графики, описывающие эволюцию интенсивности $I_{1}$ порождающего поля при трех значениях расстояния $s$, а на рис. $4, d-f$ представлены графики интенсивностей нормальных мод на тех же расстояниях.

Рисунок 4 показывает, что на достаточно большом расстоянии в области хвостовой части импульса, связанного с параллельной нормальной модой, образуется осцилляционный хвост (например, рис. 4, $c, f$ ), причем в некоторые моменты времени величина $I_{1}$ обращается в ноль. На рис. 5 представлены графики зависимости от времени величин $I_{1}$ и $\delta_{x 1}$ для нормальных мод текущего расчета на расстоянии $s=200$. Отметим, что для продольной нормальной моды выполнятся условие $\delta_{y 1}=\delta_{x 1}+\pi / 2$, тогда как для перпендикулярной моды $\delta_{y 1}=\delta_{x 1}-\pi / 2$. Согласно рис. 5 скачки функций $\delta_{x 1}$ и $\delta_{y 1}$ на величину $\pm \pi / 2$ возникают в тот момент, когда интенсивность обращается в ноль. Это означает, что в указанные моменты времени компоненты $x$ и $y$ напряженности электрического поля пробного излучения одновременно меняют знак. Подобные скачки имеются на кривой 3 , описывающей величину $\delta_{x 1}$ параллельной нормальной моды. Однако связанные с ними осцилляции интенсивности в хвостовой части этой моды настолько незначительны, что незаметны в масштабах, используемых при построении рис. 5. Результаты расчета, соглас- 

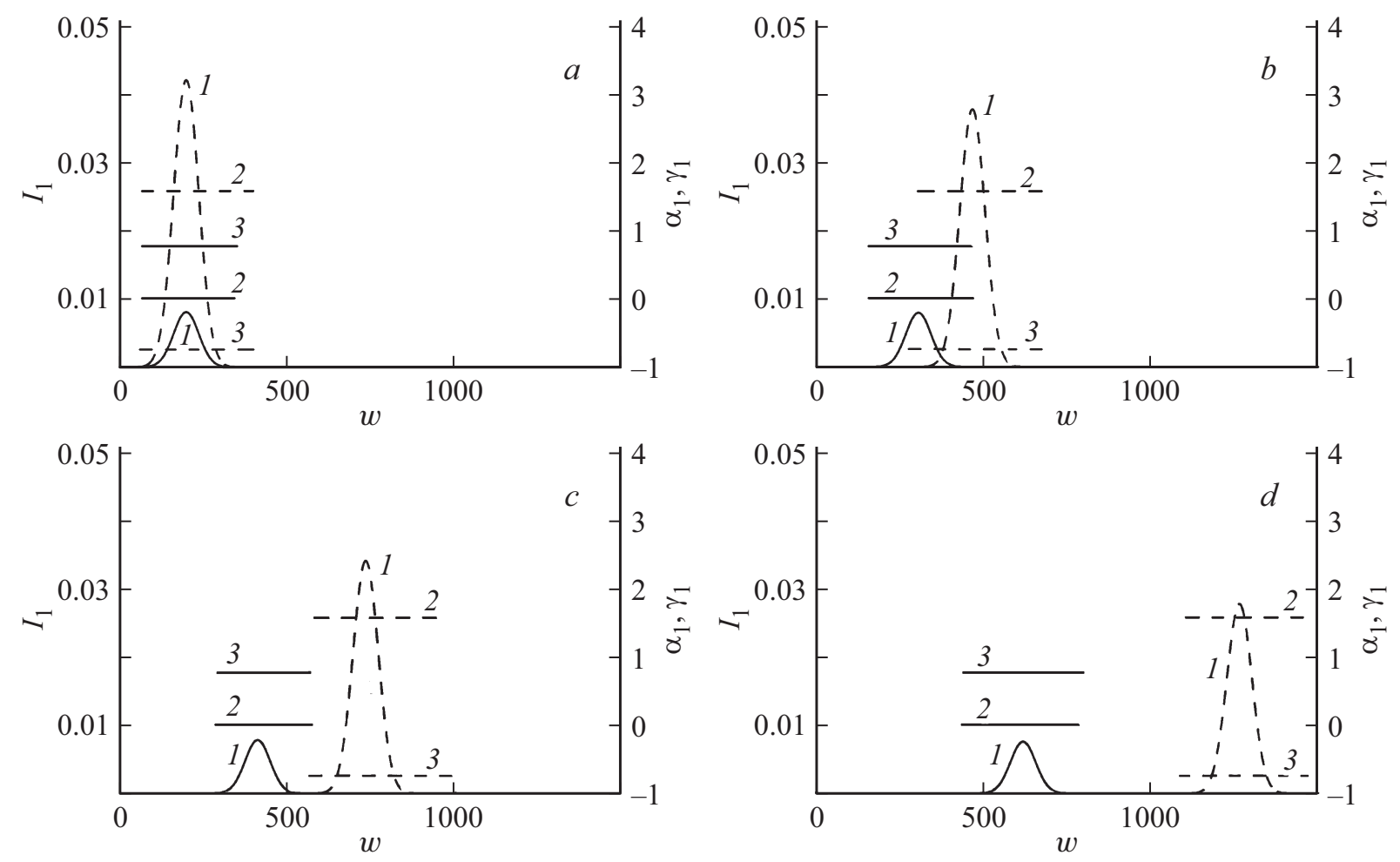

Рис. 3. Эволюция характеристик нормальных мод в среде при $s=0(a), 1000(b), 2000(c)$ и $4000(d)$; сплошные линии параллельная мода, штрихи - перпендикулярная мода; $I_{1}$ - кривые $1, \alpha_{2}$ - кривые $2, \gamma_{1}$ - кривые 3.
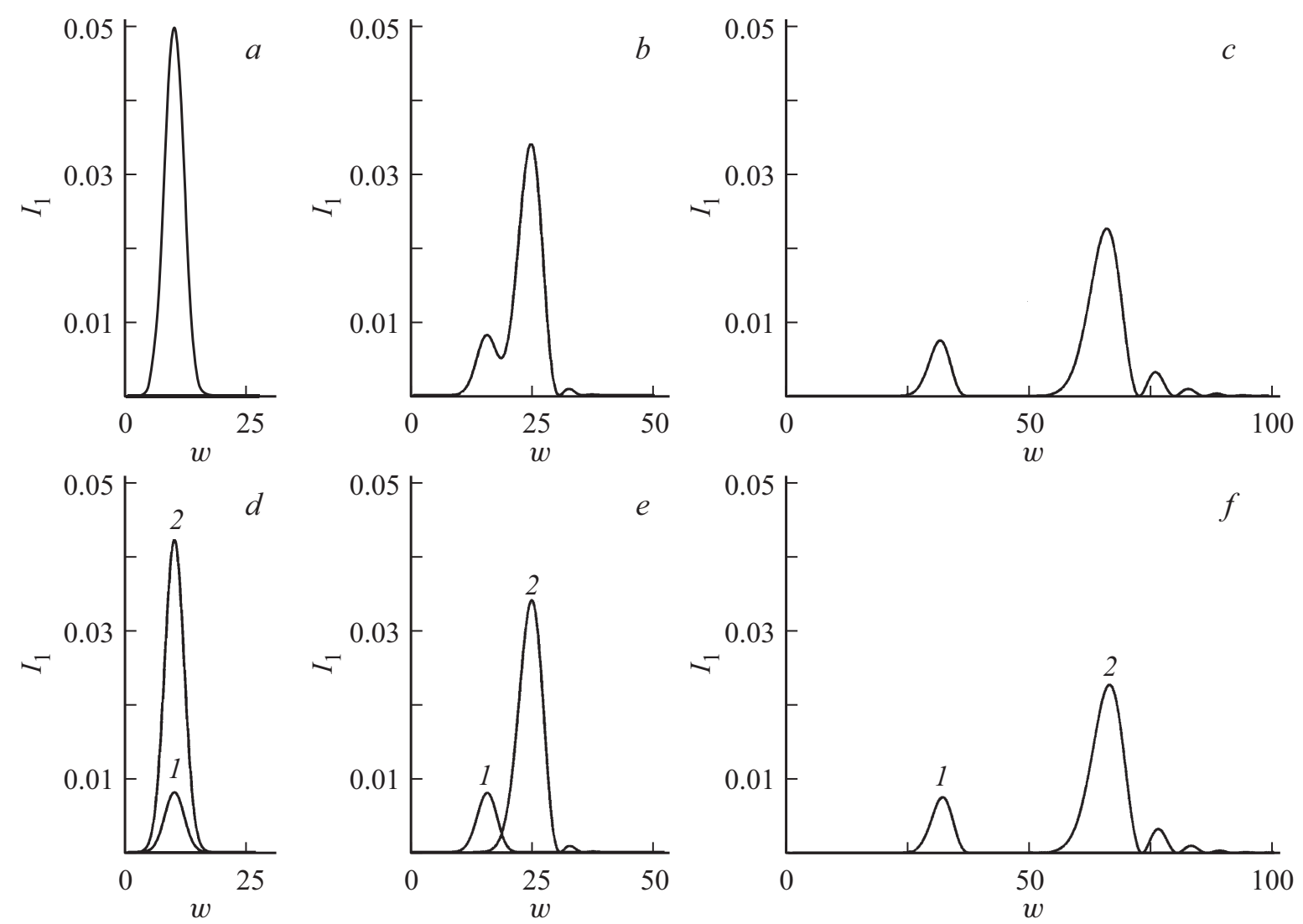

Рис. 4. Эволюция интенсивности порождающего поля при $s=0(a), 50(b), s=200(c)$, а также интенсивностей нормальных мод при $s=0(d), 50(e), 200(f) .1$ - продольная мода, 2 - перпендикулярная мода. 


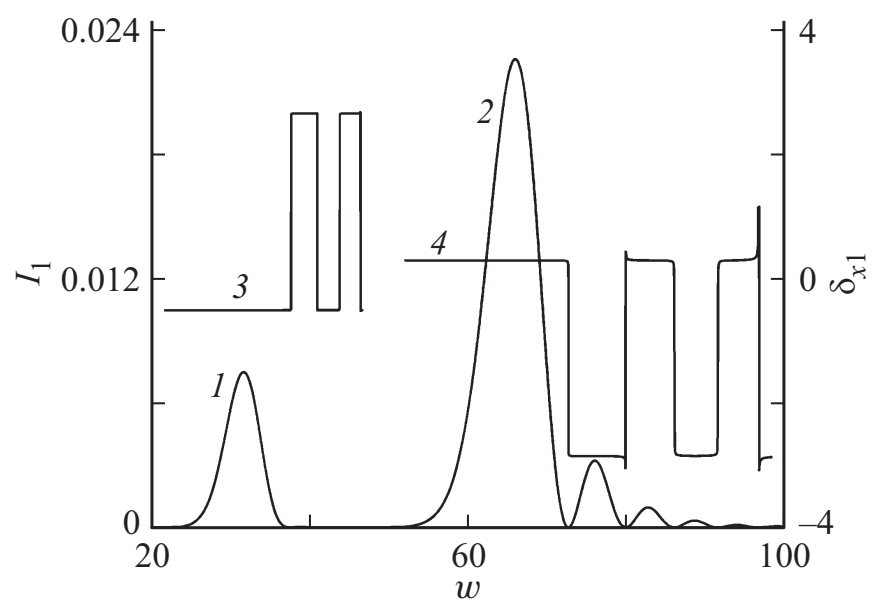

Рис. 5. Эволюция интенсивностей параллельной (1) и перпендикулярной (2) нормальных мод, а также фазовых добавок параллельной (3) и перпендикулярной (4) мод.

но которым построен рис. 4, позволяют заключить, что $V_{1}^{(1)}=9.3$ и $V_{1}^{(2)}=3.6$.

Рассмотрим случай $\tau_{p} \quad\left(T_{p}=326 \mathrm{ps}\right)$. При этом $\Delta_{p} / \Delta_{1}=2, T_{p} / \tau=0.06$. На рис. $6, a-c$ представлены графики, описывающие эволюцию интенсивности $I_{1}$ порождающего поля при трех значениях расстояния $s$, а на рис. $6, d-f$ представлены графики интенсивностей нормальных мод на тех же расстояниях. На рис. $6, d$ не изображено основание импульса 2 , чтобы был заметен импульс 1.

Рисунок 6 показывает, что излучения параллельной и перпендикулярной нормальных мод на достаточно большом расстоянии распространяются в виде довольно регулярного затухающего цуга субимпульсов. Общая длительность каждого такого цуга во много раз превосходит длительность входного пробного импульса. В момент, когда интенсивность нормальной моды обращается в ноль, происходит описанный выше скачок величин $\delta_{x}$ и $\delta_{y 1}$. Численное моделирование показало, что цуги, связанные с продольной и перпендикулярной модами, перемещаются со скоростями $V_{1}^{(1)}=8.8$ и $V_{1}^{(2)}=3.0$ соответственно.

Теперь положим, что $\tau_{p}=0.5,\left(T_{p}=163 \mathrm{ps}\right)$. Тогда $\Delta_{p} / \Delta_{1}=4, T_{p} / \tau=0.03$. На рис. 7, $a-c$ представлены графики, описывающие эволюцию интенсивности $I_{1}$ порождающего поля при трех значениях расстояния $s$, на рис. 7, $d-f$ представлены графики интенсивностей параллельной, а на рис. 7,g-i - перпендикулярной мод на тех же расстояниях. Рисунок 7 показывает, что кривые, описывающие интенсивности порождающего поля в среде, а также интенсивности образующих его нормальных мод, не имеют регулярного вида. На расстоянии $s=20$ (рис. 7, $c, f, i$ ) полная длительность действия мод пробного излучения на точку среды примерно в 30 раз превышает длительность входного пробного импульса.
В таких условиях понятие групповых скоростей нормальных мод теряет смысл.

\section{4. Заключение: обсуждение результатов}

Сравнение результатов представленных выше расчетов позволяет заключить, что с уменьшением длительности входного пробного импульса составляющие его нормальные моды приобретают черты, свойственные нестационарным процессам взаимодействия излучения со средой. В первую очередь нестационарность проявляется в существенном искажении формы огибающей импульса и разбиении импульса на фрагменты. В условиях однофотонного резонанса подобные эффекты обнаруживаются в световых нутациях, связанных с затуханием поляризации квантового перехода [29], в явлениях самоиндуцированной прозрачности [30] и фотонного эха [31]. В этих случаях проявление нестационарности становится заметным, если электромагнитное поле значительно меняется за время, равное времени необратимой релаксации квантового перехода. Последнее выполняется, когда излучение представляет собой импульс с длительностью, равной или меньшей упомянутого времени.

Однако в условиях ЭМИП влияние релаксационных процессов практически исключено [1,5]. Этим объясняется то обстоятельство, что не только в расчете с $\tau_{p}=75$, когда $T_{p} \gg \tau$, но и при $\tau_{p}=10, T_{p}=0.6 \tau$ (данный расчет выше не обсуждался).нормальные моды имеют в среде вид одиночных колоколообразных импульсов, подобных изображенным на рис. 3 .

Заметные искажения формы импульсов нормальных мод происходят при условии $\Delta_{p} / \Delta_{1} \geq 0.5$, т.е. в случае, когда спектр входного пробного импульса превосходит половину ширины спектра частот квантового перехода, резонансного пробному излучению. Об этом свидетельствуют осцилляционные всплески на заднем фронте импульса перпендикулярной нормальной моды (рис. $4, f)$.

При уменьшении длительности входного пробного импульса искажения нормальных мод в среде нарастают. Например, при $\tau_{p}=1$, когда $\Delta_{p} / \Delta_{1}=2$, нормальные моды в среде распространяются в виде достаточно длинных цугов затухающих субимпульсов (рис. 6,e,f). При еще меньших значениях длительности входного пробного импульса форма графиков интенсивности нормальных мод в среде становится хаотической (рис. $7, f, i$ ). Общая длительность импульса с такой огибающей в десятки раз превышает длительность входного пробного импульса.

Формулы (10) для групповых скоростей нормальных мод дают значения, хорошо совпадающие с полученными при численном моделировании в случае входных пробных импульсов большой длительности. Так при $\tau_{p}=75$ расхождения этих значений составляют $3 \%$ для 

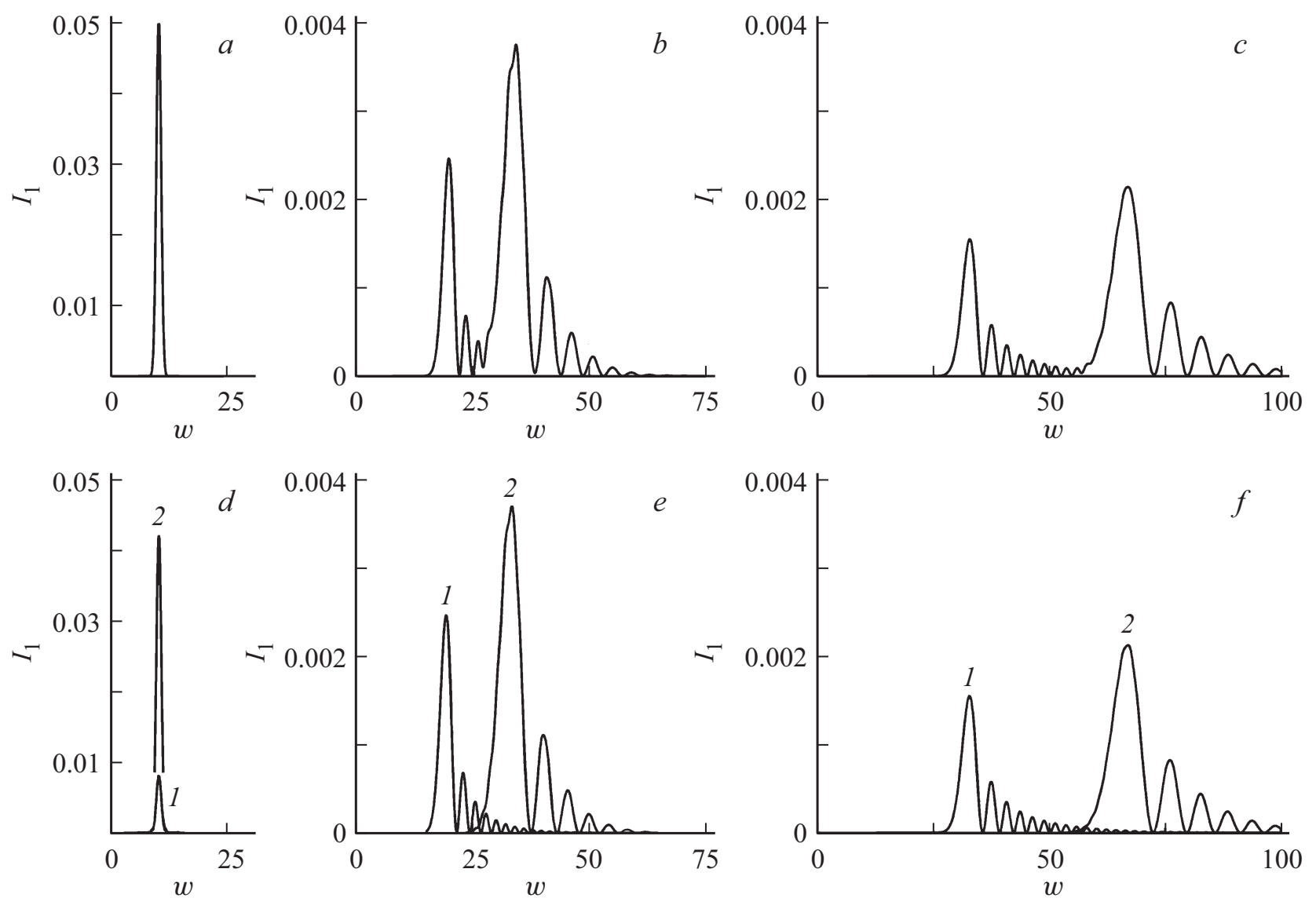

Рис. 6. Эволюция интенсивности порождающего поля при $s=0(a), 80(b), 200(c)$, а также интенсивностей нормальных мод при $s=0(d), 80(e), 200(f) .1$ - продольная мода, 2 - перпендикулярная мода.

параллельной моды и 5\% для перпендикулярной. Однако расхождения существенно возрастают при уменьшении длительности входного импульса. Так, при $\tau_{p}=1$ они равны 12 и $30 \%$ соответственно. Причина расхождений кроется в том, что вывод формул (10) основывается на нулевом порядке метода последовательных приближений, в котором деформация пробного импульса рассматривается как малая поправка (Приложение). Отметим, что именно такое предположение в различных вариантах конкретной реализации используется для введения понятия групповой скорости [32]. В случае значительной деформации импульса понятие групповой скорости как характеристики процесса его распространения теряет смысл. Именно ввиду этого выше не приводились значения групповых скоростей для случая $\tau_{p}=0.5$.

Отметим, что в случае расчета при $\tau_{p}=75$ на расстоянии $s=2000$ среда поглощает только 3\% энергии параллельной нормальной моды и 19\% перпендикулярной. В случае $\tau_{p}=0.5$ эти величины уже на расстоянии $s=20$ составляют 44 и $62 \%$ соответственно. Таким образом, с уменьшением длительности входного лазерного импульса эффективность ЭМИП существенно уменьшается. Это объясняется отклонениями от условий адиабатического следования, возникающими при достаточно быстрых изменениях поля пробного импульса [33].

\section{Приложение: групповые скорости нормальных мод}

Перейдем в системе уравнений (8) к новым независимым переменным $u$ и $v$ :

$$
u=s-V_{1}^{(1)} w, \quad v=s,
$$

где $V_{1}^{(1)}$ - действительная постоянная. Предположим, что справедливы следующие равенства:

$$
\begin{gathered}
g_{1}^{(1)}=\bar{g}_{1}^{(1)}(u)+\varepsilon \tilde{g}_{1}^{(1)}(u, v), \quad P_{1}=\bar{P}_{1}(u)+\varepsilon \tilde{P}_{1}(u, v), \\
Q_{1}=\bar{Q}_{1}(u)+\varepsilon \tilde{Q}_{1}(u, v),
\end{gathered}
$$

где $\varepsilon$ - малый параметр, так что вторые слагаемые в правых частях последних равенств являются малыми поправками, влияние которых можно учесть методом последовательных приближений. В нулевом порядке этого метода $g_{1}^{(1)}=\bar{g}_{1}^{(1)}(u), P_{1}=\bar{P}_{1}(u)$ и $Q_{1}=\bar{Q}_{1}(u)$. Действительные и мнимые части этих функций описывают возмущения, перемещающиеся в среде без изменения 

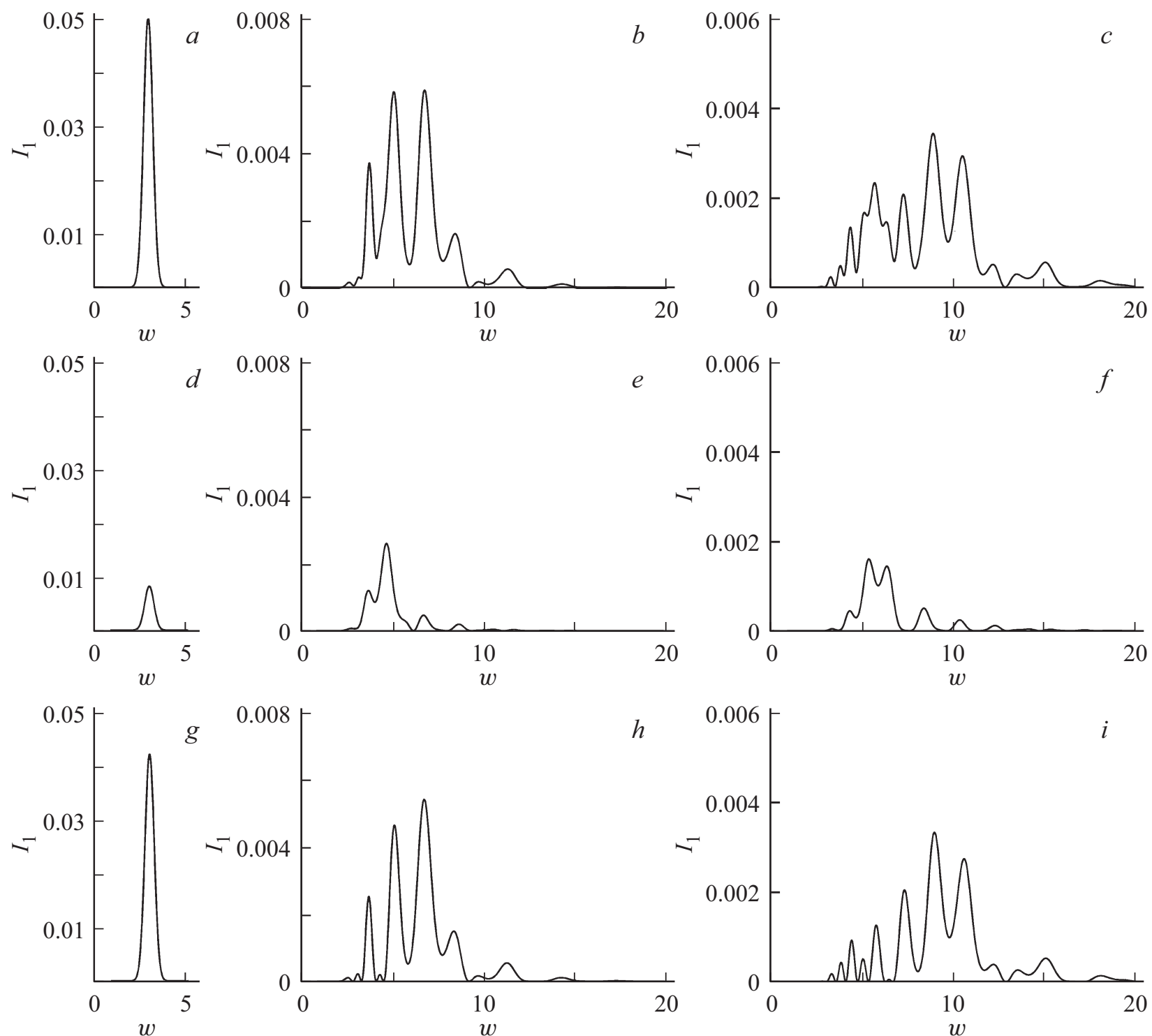

Рис. 7. Эволюция интенсивности порождающего поля при $s=0(a), 10(b), 20(c)$, интенсивности параллельной нормальной моды при $s=0(d), 10(e), 20(f)$ и интенсивности перпендикулярной моды при $s=0(g), 10(h), 20(i)$.

формы со скоростью $V_{1}^{(1)}$, которую принято называть групповой скоростью.

Сохраняя только члены нулевого порядка по малому параметру $\varepsilon$, приведем систему уравнений (8) к следующему виду:

$$
\begin{gathered}
\frac{\partial \bar{g}_{1}^{(1)}}{\partial u}=\frac{2 i}{\sqrt{\pi}} \int_{-\infty}^{+\infty} \bar{U}_{1}^{*} \exp \left(-\varepsilon_{1}^{2}\right) d \varepsilon_{1} \\
\frac{d \bar{U}_{1}}{d u}=i\left(\frac{\varepsilon_{1}}{V_{1}^{(1)}}\right) \bar{U}_{1}+i\left(\frac{g_{20}^{*}}{4 V_{1}^{(1)}}\right) \bar{V}_{1}+i\left(\frac{\bar{g}_{1}^{*}}{2 V_{1}^{(1)}}\right) \\
\frac{d \bar{V}_{1}}{d u}=i\left(\frac{g_{20} q_{1}}{V_{1}^{(1)}}\right) \bar{U}_{1}-i\left(\frac{\alpha \varepsilon_{1}}{V_{1}^{(1)}}\right) \bar{V}_{1}
\end{gathered}
$$

где $\alpha=2-\omega_{2} / \omega_{1}$. Записывая систему (П1), мы опустили слагаемое, учитывающее необратимую релаксацию. Его учет не меняет конечного результата.

Введем величины $\Theta_{1}$ и $\Theta_{2}$ следующим образом:

$$
\begin{aligned}
& \Theta_{1}=\frac{1}{\sqrt{\pi}} \int_{-\infty}^{+\infty} \bar{U}_{1}^{*} \exp \left(-\varepsilon_{1}^{2}\right) d \varepsilon_{1}, \\
& \Theta_{2}=\frac{1}{\sqrt{\pi}} \int_{-\infty}^{+\infty} \bar{V}_{1}^{*} \exp \left(-\varepsilon_{1}^{2}\right) d \varepsilon_{1} .
\end{aligned}
$$

Умножая обе части последних двух уравнений системы (П1) на $(1 / \sqrt{\pi}) \exp \left(-\varepsilon_{1}^{2}\right)$ и интегрируя полученные 
соотношения по $\varepsilon_{1}$ в пределах от $-\infty$ до $+\infty$, получаем

$$
\begin{gathered}
\frac{d \bar{g}_{1}^{(1)}}{d u}=2 i \Theta_{1}, \\
\frac{d}{d u} \Theta_{1}=-i \frac{g_{20}}{4 V_{1}^{(1)}} \Theta_{2}-i \frac{\bar{g}_{1}^{(1)}}{2 V_{1}^{(1)}}-i \frac{1}{V_{1}^{(1)}} F_{1}, \\
\frac{d}{d u} \Theta_{2}=-i \frac{g_{20} q_{1}}{V_{1}^{(1)}} \Theta_{1}-i \frac{\alpha}{V_{1}^{(1)}} F_{2},
\end{gathered}
$$

где величины $F_{1}$ и $F_{2}$, входящие в правые части уравнений (П3) и (П4), определяются формулами

$$
\begin{aligned}
& F_{1}=\frac{1}{\sqrt{\pi}} \int_{-\infty}^{+\infty} \varepsilon_{1} \bar{U}_{1}^{*} \exp \left(-\varepsilon_{1}^{2}\right) d \varepsilon_{1} \\
& F_{2}=\frac{1}{\sqrt{\pi}} \int_{-\infty}^{+\infty} \varepsilon_{1} \bar{V}_{1}^{*} \exp \left(-\varepsilon_{1}^{2}\right) d \varepsilon_{1}
\end{aligned}
$$

Комбинируя уравнения (П3) и (П4), получаем

$$
\begin{gathered}
\frac{d^{2}}{d u^{2}} \Theta_{1}+k \Theta_{1}=F_{0}, \\
F_{0}=\frac{1}{V_{1}^{(1)}} \frac{d}{d u} F_{1}-\frac{\alpha g_{20}}{4 V_{1}^{(1)}} F_{2}, \\
k=\frac{1}{V_{1}^{(1)}}\left(\frac{\left|g_{20}\right|^{2} q_{1}}{4 V_{1}^{(1)}}-1\right) .
\end{gathered}
$$

Величина $\Theta_{1}$ согласно уравнению (П2), представляет собой поляризационный источник, под воздействием которого эволюционирует поле пробного излучения. Будем рассматривать функцию $F_{0}$ в правой части уравнения (П5) как неоднородный член. Тогда общее решение уравнения (П5) будет суммой решений однородного и неоднородного уравнений. В случае $k>0$ однородное уравнение имеет периодические решения. При $k<0$ решения однородного уравнения являются неограниченными по модулю на $-\infty$ или на $+\infty$. Наличие решений с такими свойствами не способствует распространению пробного излучения в виде импульсов - порций энергии поля, затухающих при $u \rightarrow \pm \infty$. Поэтому сделаем предположение, что выполняется равенство $k=0$. Используя (П6), получаем тогда

$$
V_{1}^{(1)}=(1 / 4)\left|g_{20}\right|^{2} q_{1} .
$$

Выражая $g_{20}$ через характеристики управляющего излучения, получаем формулу (10) при $i=1$. Аналогично обосновывается формула (10) в случае $i=2$. Сделанное выше предположение является эвристическим, и степень его адекватности проверяется изложенными в основном тексте статьи результатами численного моделирования.

\section{Список литературы}

[1] Агапьев Б.Д., Горный М.Б., Матисов Б.Г., Рождественский Ю.В. // УФН. 1993. Т. 163. № 9. С. 1; Agap'ev B.D., Gornyi M.B., Matisov B.G., Rozhdestvenskii Yu.V. // PhysicsUspekhi 1993. V. 36. N 9. P. 763.

[2] Vitanov N.V., Rangelov A.A., Shore B.W., Bergmann K. // Rev. Mod. Phys. 2017. V. 89. N 1. P. 015006.

[3] Harris S.E. // Phys. Today. 1997. V. 50. N 7. P. 36.

[4] Lukin M.D. // Rev. Mod. Phys. 2003. V. 75. N 2. P. 457.

[5] Fleischhauer M., Imamoğlu A., Marangos J.P. // Rev. Mod. Phys. 2005. V. 77. N 2. P. 633.

[6] Duan L.-M., Lukin M.D., Cirac J.I., Zoller P. // Nature (London). 2001. V. 414. P. 413.

[7] Sinatra A. // Phys. Rev. Lett. 2006. V. 97. N 25. P. 253601.

[8] Martinalli M., Valente P., Failache H., Felinto D., Cruz L.S., Nussenzveig P., Lezama A. // Phys. Rev. A. 2004. V. 69. N 4. P. 043809.

[9] Godone A., Micallilizio S., Levi F. // Phys. Rev. A. 2002. V. 66. N 6. P. 063807.

[10] Lukin M.D., Imamoğlu A. // Nature (London). 2001. V. 413. P. 273.

[11] Kocharovskaya O., Mandel P. // Phys. Rev. A. 1990. V. 42. N 1. P. 523.

[12] Jen H.H., Daw-Wei Wang. // Phys. Rev. A. 2013. V. 87. N 6. P. 061802(R).

[13] Basler C., Grzesiak J., Helm H. // Phys. Rev. A. 2015. V. 92. N 1. P. 013809.

[14] Ronggang Liu, Tong Liu, Yingying Wang, Yujie Li, Bingzheng Gai. // Phys. Rev. A. 2017. V. 96. N 5. P. 053823.

[15] Fam Le Kien, Rauschenbeutel A. // Phys. Rev. A. 2015. V. 91. N 5. P. 053847.

[16] Wielandy S., Gaeta A.L. // Phys. Rev. Lett. 1998. V. 81. N 16. P. 3359.

[17] Bo Wang, Shujing Li, Jie Ma, Hai Wang, Peng K.C., Min Xiao. // Phys. Rev. A. 2006. V. 73. N 5. P. 051801(R).

[18] Agarwal G.S., Shubhrangshu Dosgupta.// Phys. Rev. A. 2003. V. 67. N 2. P. 023814.

[19] Sautenkov V.A., Rostovtsev Y.V., Chen H., Hsu P., Agarwal G.S., Scully M.O. // Phys. Rev. Lett. 2005. V. 94. N 23. P. 233601.

[20] Tai Hyun Yoon, Chang Yong Park, Sung Jong Park. // Phys. Rev. A. 2004. V. 70. N 6. P. $061803($ R).

[21] Паршков О.М. // Опт. и спектр. 2017. Т. 123. № 3. С. 405; Parshkov O.M. // Opt. Spectrosc. 2017. V. 123. N 3. P. 430.

[22] Париков О.М. // Квант. электрон. 2018 Т. 48. № 11. C. 1027; Parshkov O.M. // Quant. Electron. 2018. V. 48. N 11. P. 1027.

[23] Kasapi A., Maneesh Jain, Yin G.Y., Harris S.E. // Phys. Rev. Lett. 1995. V. 74. N 13. P. 2447.

[24] Maneesh Jain, Kasapi A., Yin G.Y., Harris S.E. // Phys. Rev. Lett. 1995. V. 75. N 24. P. 4385.

[25] Saleh B.A.E., Teich M.C. Fundamentals of photonics, 2th ed. Wiley-Interscience, 2007. 1177 р. Перевод: Салех Б., Teŭx M. Оптика и фотоника. Принципы и применения. Долгопрудный: ИД „Интеллект“, 2012. Т. 1. 760 с.

[26] Борн М., Вольф Э. Основы оптики. М.: Наука, 1970. 855 с. Born M., Wolf M. Principles of optics, 7th. Cambridge University Press, 1999. 987 p.

[27] deZafra R.L., Marshall A. // Phys. Rev. 1968. V. 170. N 1. P. 28. 
[28] Физические величины. Справочник / Под. ред. Григорьева И.С., Мейлихова Е.3. М.: Энергоатомиздат, 1991. $1232 \mathrm{c}$.

[29] Акулин В.М., Карлов М.В. Интенсивные резонансные взаимодействия в квантовой электронике. М.: Наука, 1987. $312 \mathrm{c}$.

[30] McCall S.L., Hahn E.L. // Phys. Rev. 1969. V. 183. N 2. P. 457.

[31] Евсеев И.В., Рубцова Н.Н., Самарцев В.В. Когерентные переходные процессы в оптике. М: Физматлит, 2009. 536 с.

[32] Виноградова М.Б., Руденко О.В., Сухоруков А.П. Теория волн. М.: Наука, 1990. 432 с.

[33] Kozlov V.V., Kozlova E.B. // Opt. Commun. 2009. V. 282. N 5.

P. 892. 